\title{
LA RELACIÓN ENTRE LA COMUNICACIÓN INTERNA Y EL CLIMA LABORAL EN LOS DOCENTES DE LA UNIDAD EDUCATIVA SAN JOAQUÍN, DE LA PARROQUIA CUMBE, EN EL PERIODO 2018
}

\section{THE RELATIONSHIP BETWEEN INTERNAL COMMUNICATION AND LABOR CLIMATE IN THE TEACHERS OF THE SAN JOAQUÍN EDUCATIONAL UNIT, OF THE CUMBE PARISH, IN THE PERIOD 2018}

\author{
Autor: Henry Patricio Iñaguazo Macas \\ E-mail de contacto: ihenrypatricio@yahoo.com \\ Articulo recibido: 31 de Septiembre del 2019 \\ Articulo revisado: 4 de Noviembre del 2019 \\ Articulo aprobado: 21 de Diciembre del 2019 \\ Licenciado en Ciencias de la Educación mención Informatica egresado de la Universidad de Guayaquil (Ecuador) con 6 \\ años de experiencias en la docencia. Maestrante en la Universidad Nacional Mayor de San Marcos de Lima (Perú), mención \\ Gestión de la Educación.
}

\section{Resumen}

Para el desarrollo del país, para el trabajo en las empresas o instituciones, la comunicación es un axial. Las estrategias de comunicación dentro de una organización garantizan la productividad y un excelente clima laboral. Por ello, en la presente investigación se analiza la relación entre la comunicación interna y el clima laboral en los docentes de la unidad educativa San Joaquín, de la Parroquia Cumbe, en el periodo 2018. Esta investigación es de enfoque cuantitativo, alcance correlacional, tipo no experimental, de diseño transeccional, de temporalidad transversal. Se aplicó dos encuestas a 30 docentes de la Unidad Educativa San Joaquín, de la Parroquia Cumbe, en el periodo 2018. Los cuestionarios corresponden a una variable: comunicación interna y clima laboral. El primer instrumento está compuesto por 34 ítems divididos en tres dimensiones: patrones de comunicación, barreras de comunicación y retroalimentación. Mientras que el cuestionario sobre el clima laboral está constituido por 25 ítems divididos en cinco dimensiones: liderazgo, relaciones interpersonales, participación, reconocimiento y comunicación. En la investigación se concluyó que el $30 \%$ considera que "A veces" se cumple con el clima laboral y la comunicación interna. Mientras que el 53,3\% considera "casi siempre" se establece la relación en ambas variables. Según la prueba de hipótesis, se concluye que existe una relación entre moderada y fuerte con significación estadística. Esta correlación es positiva, es decir a mayor frecuencia con respecto a la comunicación interna, mayor satisfacción habrá en el clima laboral. $(\mathrm{p}<0.05)$

Palabras claves: Comunicación Interna, Clima Laboral, Calidad Educativa, Gestión Educativa.

\section{Abstract}

For the development of the country, for work in companies or institutions, communication is axial. Communication strategies within an organization guarantee productivity and an excellent work environment. Therefore, this research analyzes the relationship between internal communication and the work environment in teachers of the San Joaquin educational unit, of Cumbe Parish, in the 2018 period. This research is quantitative, correlational, type scope non-experimental, transectional design, transverse temporality. Two surveys were applied to 30 teachers of the San Joaquín Educational Unit, of the Cumbe, in the 2018 period. The questionnaires correspond to a variable: internal communication and work environment. The first instrument is made up of 34 items divided into three dimensions: communication patterns, communication barriers and feedback. While the questionnaire on the work environment is constituted by 25 items divided into five dimensions: leadership, interpersonal relationships, participation, recognition and communication. In the investigation it was concluded that $30 \%$ 


\section{Ciencia y Educación (ISSN 2707-3378) \\ Vol. 1 No. 2 \\ Febrero del 2020}

Como componente de la dimensión de Gestión administrativa, la información y comunicación "es el conjunto de acciones y recursos tecnológicos que facilitan el acceso a la información y la comunicación intra e interinstitucional, para fortalecer el proceso de enseñanza aprendizaje" (Ministerio de Educación, 2017, p.15). Por ello, en la presente investigación se buscará establecer la relación que existe entre la comunicación interna se relaciona con el clima laboral de los docentes de la Unidad Educativa San Joaquín, de la Parroquia Cumbe, en el periodo 2018.

Por ello, la investigación plantea como problema general ¿En qué medida la comunicación interna se relaciona con el clima laboral de los docentes de la Unidad Educativa San Joaquín, de la Parroquia Cumbe, en el periodo 2018?

Asimismo, la importancia del presente estudio sobre la comunicación interna y su relación con el clima laboral se sustenta en una necesidad contextual y académica. En relación con lo contextual, es importante analizar esta relación para mejorar la relación laboral en las instituciones educativas del país en el marco de la calidad educativa.

Ante esta problemática, el estudio se propone como objetivo: Establecer la relación que existe entre la comunicación interna se relaciona con el clima laboral de los docentes de la Unidad Educativa San Joaquín, de la Parroquia Cumbe, en el periodo 2018. Asimismo, pretende establecer la relación que existe entre el área de patrones de comunicación, el área de barreras de la comunicación el área de retroalimentación de la comunicación interna se relaciona con el clima laboral de la de la Unidad Educativa San Joaquín, de la Parroquia Cumbe, en el periodo 2018.
La hipótesis de investigación se formula a partir de aceptar que existe relación significativa entre la comunicación interna y el clima laboral de los docentes de la Unidad Educativa San Joaquín, de la Parroquia Cumbe, en el periodo 2018.

En la actualidad, la comunicación es analizada por el impacto, por ejemplo se conceptualiza sobre una comunicación de masas, es decir, una comunicación es "caracterizada por la distribución masiva de un mensaje unidireccional de uno a muchos" (Wright, 1995, p.14). Sin embargo, con el surgimiento de la web 2.0, la comunicación se ha concedido como horizontal. Para Ramos (2015),

La comunicación horizontal ha sido posible debido a la expansión de Internet, la web 2.0, los medios digitales, la comunicación móvil, la gran variedad de herramientas de software, las cuales son la base para las redes sociales y han dado lugar a una comunicación interactiva que permite conectar local y globalmente en un tiempo determinado a los individuos. (p.30)

Los modos de comunicación, también, se establecen en el ámbito empresarial. Para WillsEsponisa, Cevallos, Sadi y Ancin (2017), en su breve historia de la comunicación organizacional, considera que esta se origina en EE. UU. hacia finales de 1930 y comienzos de 1940, aunque la cima se generará en la década de 1970 con los trabajos de Wioo (1976) y Downs y Hazen (1977).

La investigación de Downs y Hazen (1977) reveló la naturaleza multidimensional de la comunicación organizacional, lo cual significa que los empleados pueden sentirse satisfechos en distintos grados sobre categorías específicas de la comunicación. Actualmente el instrumento Cuestionario de la Satisfacción con la Comunicación (CSQ por sus siglas en inglés) 
mide diez factores sobre el funcionamiento de la comunicación y su satisfacción:

- Calidad de los medios,

- El clima organizacional,

- Comunicación con el supervisor,

- Comunicación con los subordinados,

- Comunicación horizontal e informal,

- Integración organizacional,

- Perspectiva de la organización,

- Retroalimentación personal,

- Comunicación de los altos directivos

- Comunicación interdepartamental.

La comunicación organizacional se clasificará según los modos en cinco procedimientos: Círculo, rueda, cadena, estrella y total. (ver Figura 1)

Figura 1: Modos de comunicación en las organizaciones

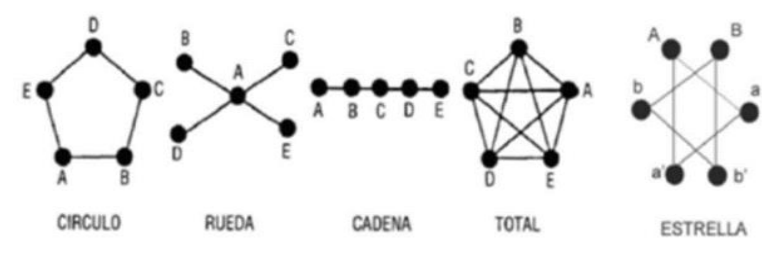

Nota: Charry (2017)

El estudio de esta problemática ha sido desarrollado Osorio (2009) y Romero (2013) quienes destacan la importancia de la comunicación interna en el mayor impacto y repercusión posible. Asimismo, Baladezo (2007) haya evidencias que destacan la importancia de la calidad de la comunicación organizacionacional con la productividad y con las actitudes de los trabajadores.

Por su parte, Cabrera (2012) concluye que existen marcadas diferencias de opinión entre los directivos, docentes y estudiantes en la forma como se ha manejado la comunicación al interior de una organización, por lo que urgen identificar los públicos internos de tal manera que se presenten estrategias particulares según las características de cada actor. Roca (2012) advierte sobre los peligros que se presentan cuando una organización tiene resultados negativos respecto a su calidad de comunicación interna y Reyes (2017) concluye que incluso al interior de cada dimensión estudiada puede haber variaciones que deben ser atendidas

Es necesario entender la comunicación como un proceso que se encarga de afianzar los lazos entre los individuos, permite expresar ideas y sentimientos; por ello, los integrantes de un grupo social deben comunicar sus necesidades y requerimientos para llegar a la solución de los problemas. Bajo este marco, Duque (2005, p. 15) afirma que la comunicación "es un elemento básico de toda interacción humana, ya sea entre individuos, de un individuo con un grupo o entre diferentes subgrupos de personas".

Desarrollado el flujo comunicativo, según Kuss (2015), se clasifican en dos tipos de comunicación: externas e internas. Las primeras se caracterizan por tratarse del flujo de la comunicación entre la empresa y sus clientes, de tal modo que se inicia la comunicación hacia el exterior:

"[...] va dirigida al público externo: clientes, intermediarios, proveedores, competencia, medios de comunicación y público en general. Viene determinada por las personas que no tienen ninguna relación con la empresa o bien la tienen de manera muy limitada, sea esta geográfica, de productos o de servicio. Aunque está vinculada a departamentos tales como Gabinetes de Prensa o de Relaciones Publicas o al de Comunicación Corporativa, todos los, miembros de la organización pueden realizar funciones de comunicación externa y de difusión de la propia imagen de la organización. Dentro de ella también se incluyen las campañas de marketing y publicidad." (Vértice, 2007, p. 6). 


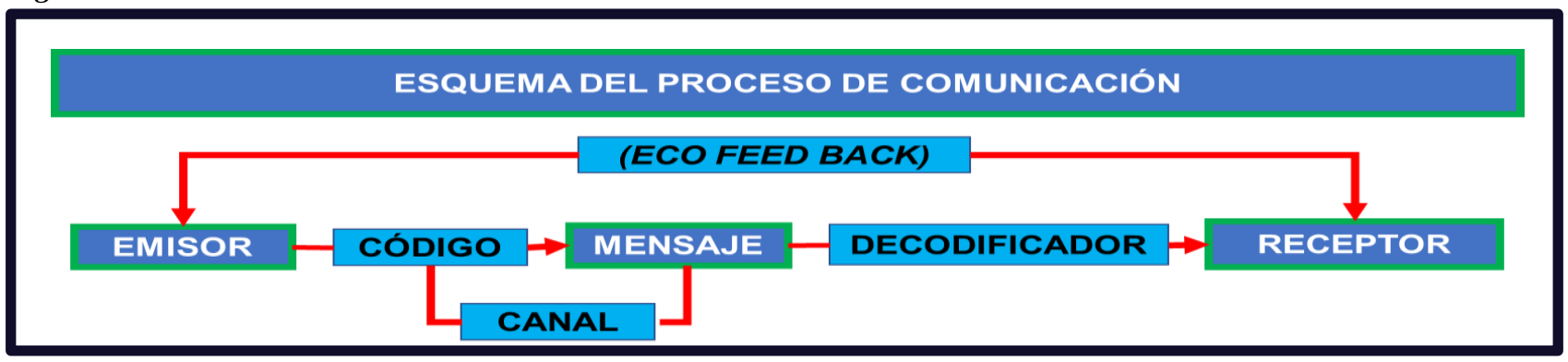

Nota: Fernández y Fernández (2010)

Según Fernández y Fernández (2010) se presentan seis elementos de la comunicación: emisor, código, mensaje, decodificador, receptor y canal (Figura 2)

La comunicación interna, por otro lado, es el proceso comunicativo que se da dentro de una organización, es decir, el diálogo que se establece entre los trabajadores y directivos de una empresa. Además, la comunicación interna está "relacionada con contar a todos los miembros de una organización lo que ésta hace $\mathrm{y}$, sobre todo, contar con los miembros de la organización para lo que ésta hace." (Arizcure, Cabezas, Cañeque, Casado, Fernández, Lacasta y Soria, 2008, p. 18).

Si la comunicación interna es exitosa, la empresa tendrá una mejor organización entre sus empleados, mejorando la productividad; de modo contrario ocurriría que la empresa no logre la integración de sus miembros propiciando la baja productividad

En la actualidad, según Arizcure, et al. (2011), comunicación interna es un elemento cuantificable que logra mejorar las labores dentro de las organizaciones ya que está considerada como una herramienta muy eficaz para la gestión de las organizaciones, debido a que se puede utilizar para analizar la realidad concreta que existe en ella, una realidad que puede ser medible de forma objetiva, mediante cuestionarios y escalas de valoración, o de formas más subjetiva a través de entrevistas, conversaciones o estudios de la documentación interna que circula por toda la empresa." (p. 34).

La comunicación en las organizaciones involucra a todo el personal, y cada persona presenta un sistema cognitivo distinto, de tal manera que la información que salió del emisor no llegue con la intención o con el porcentaje total de lo que quiso transmitir, es decir, los receptores captaran el mensaje de forma distinta, lo que va a propiciar que cada uno actué según a lo que comprendió.

Sin embargo, es fundamental lo señalado, debido a que, si hablamos de una orden gerencial, no será acatada de forma igualitaria por los trabajadores y ello hará que el fin común hacia el cual marchaba la organización cambie de rumbo o nunca llegue a él. En conclusión, la comunicación en las organizaciones es de dos tipos internas y externas, siendo fundamental en las organizaciones la comunicación interna (Figura 4.) y depende de cómo sea la relación entre emisor y receptor se pueden establecer diversos tipos de comunicación dentro de las organizaciones.

La comunicación interna dentro de las empresas $\mathrm{u}$ organizaciones presentan diferentes características, de acuerdo con Arizcuren et al. (2008, pp.24-25) se dividen en dos grupos: Formal e Informal. La primera se caracteriza por ser planificada y estructurada, 


\section{Ciencia y Educación (ISSN 2707-3378) \\ Vol. 1 No. 2 \\ Febrero del 2020}

La comunicación informal, por otro lado, es aquella que se suscita a través de medios ajenos a los empleados por la empresa, es decir, puede ocurrir entre los trabajadores dentro como fuera de la empresa y pueden ser conversaciones en sobremesa, por medios electrónicos o simples conversaciones de pasillo.

Para que una organización pueda realizar satisfactoriamente la comunicación interna es necesario que se guie de algunos procedimientos, los cuales deben ser ejecutados de forma ordenada y precisa para lograr los resultados esperados. Según Reyes (2012, pp. 131-136), la comunicación interna presenta cuatro dimensiones: vínculo, afectividad, orgullo e identidad.

Álvarez (2011), tras el aporte de Michael Ritter (2008) en su libro Cultura Organizacional, señala que la medición de los resultados, de la comunicación interna, solo pueden ser evaluados bajo el aumento de o mejoramiento de términos, también, intangibles. Además, el clima laboral repercute en la comunicación interna ya que involucra a toda la organización y a los diferentes niveles organizacionales.

Álvarez (2011, pp. 81-92) señala la estadística de repercusión de la comunicación interna dentro de la empresa, muestra diferentes casos donde en todos se hace primordial establecer a hacia donde se aspira llegar, del mismo modo los empleados señalan que el papel de la medición es un aporte significativo para evaluar los resultados.

La Comunicación interna dentro de la organización tiene como principal finalidad mejorar la gestión directiva, porque involucra a todo el personal hacia los objetivos trazados por la empresa, cohesiona al grupo de trabajo, mejora el clima laboral y se ven reflejados en la productividad. Con ello "la comunicación interna es pues, un interesante instrumento de gestión de RR.HH., y se integra en el contexto de las políticas de personal." (Equipo Editorial, 2007, p. 8)

El clima laboral es la asociación que se da entre los empleados y el ambiente organizacional, en el cual se logra hacer la diferencia entre las empresas; como es lógico, cada organización se conforma y presenta objetivos distintos; por ello, se debe analizar cada uno de los factores que intervienen.

Para Govea, Domínguez y San Agustín (2012) se presentan cuatro factores:

- Aspectos psicológicos: Son las necesidades que el personal establece, así como los procesos emocionales que genera hacia la empresa y en las relaciones horizontales. La percepción que tiene el empleado hacia la empresa se va a ver refleja en como actué dentro de esta.

- Cultural: Está referido a la interacción éntrelos individuos que involucra el modo de vida, manifestaciones e interacción, por ello es relevante dentro de las organizaciones, ya que cada persona presenta idiosincrasias disímiles que terminan reflejándose en el ambiente laboral.

- Situaciones Laborales: Son las que se generan a partir de la interacción entre el personal y que dependerán del ambiente de trabajo.

- Entorno social: Es la influencia que la sociedad ejerce sobre las empresas y como estas se proyectan a impactar a la sociedad. pp. (2-4).

Álvarez (2006), también, tiene en cuenta a Friedlander y Margulies (1969), señala que el 


\section{Ciencia y Educación (ISSN 2707-3378) \\ Vol. 1 No. 2 \\ Febrero del 2020}

clima laboral es un fenómeno índole social y personal, es decir, que la sociedad influye sobre la perspectiva del trabajador. Considera que se plasman ocho dimensiones como: Empeño, Obstáculos, Intimidad, Espíritu de trabajo. Actitud, Acento puesto sobre la producción, Confianza, Consideración (p.55)

El desempeño laboral es la capacidad de respuesta que demuestra el trabajador frente a las labores que se le encomiendan. Generalmente se relaciona la capacidad de respuesta con el nivel de inteligencia que presenta el asalariado; sin embargo, ello se ha demostrado como erróneo, debido a que psicológicamente existen otros procesos, tales como la creatividad, asertividad, liderazgo, etc.

Las dimensiones empleadas por Litwin y Stringer, por ejemplo, consideran situaciones en las cuales atraviesa el trabajador con referente a sus motivaciones para conseguir el éxito; Sims y Lafollette toman en cuenta la satisfacción del personal, al igual que Pritchard y Karasick. De este modo, se pueden destacar tres factores generales que inciden en el desempeño laboral:

- Capacitación: Es el proceso que dota de conocimientos específicos al trabajador sobre las funciones que debe realizar y como puede incrementar sus resultados. Pérez (2011, p. 6) señala que la capacitación se da "[...] como el desarrollo de habilidades específicas para el trabajo y $[\ldots]$ el desarrollo de competencias laborales [...]", es decir, las capacitaciones incrementan la productividad.

- Motivación e incentivo: La motivación es el impulso que motiva al asalariado a realizar su trabajo, generalmente las motivaciones están entre niveles familiares $y$ de realización personal. Por otro lado, el mejor impulso dentro de las organizaciones, son los incentivos que brinda la empresa, ello promueve y desarrolla la competitividad entre los trabajadores; de este modo llevan a cabo "una acción ya sea de orden colectivo, político-institucional o de carácter individual, que posibiliten un cambio cualitativo al interior de una institución. [...] [y los incentivos] se pueden clasificar en monetarios y no monetarios." (Elias, 2004, p. 9).

- Eficiencia y Eficacia: Primero la eficiencia es la "Capacidad de disponer de alguien o de algo para conseguir un efecto determinado" y eficacia es la "Capacidad de lograr el efecto que se desea o se espera.". Dentro de una organización, los trabajadores deben disponer, de la mejor manera, los instrumentos que se les brinda para realizar su función y debe demostrar eficacia en el cumplimiento de dicha meta.

\section{Metodología de la Investigación}

El tipo y Diseño de Investigación se realiza en base a la taxonomía planteada por Hernández Sampieri (2014): El enfoque de la investigación es cuantitativa, el alcance es correlacional, el tipo es no experimental, el diseño de es transeccional correlacional y la temporalidad: transversal

La unidad de análisis se realizará en la Unidad Educativa San Joaquín, de la Parroquia Cumbe, en el periodo 2018, cuya población de estudio está constituida por los 60 docentes de la Unidad Educativa San Joaquín, de la Parroquia Cumbe, Cantón Cuenca, provincia de Azuay. 


\section{Ciencia y Educación (ISSN 2707-3378) \\ Vol. 1 No. 2 \\ Febrero del 2020}

Según Hernández Sampieri (2014), la selección de la muestra se puede realizar de acuerdo al propósito del estudio como la investigación requiere un análisis estadístico, el tamaño mínimo de muestra por tipo de análisis comparativo en diseños experimentales será de 30 casos.

Para analizar la relación entre la comunicación interna y el clima laboral se ha utilizado dos cuestionarios. Estos instrumentos posibilitan cuantificar la percepción de los docentes. El cuestionario sobre la motivación intrínseca está constituido por 34 ítems divididos en tres dimensiones: patrones de comunicación, barreras de comunicación y retroalimentación.

El cuestionario sobre el clima laboral está constituido por 25 ítems divididos en cinco dimensiones: liderazgo, relaciones interpersonales, participación, reconocimiento y comunicación.

\section{Resultados y discusión}

En cuanto al género de los docentes de la institución educativa San Joaquín, del total de 30 encuestados, 15 corresponde al sexo masculino y 15 con del sexo femenino En cuanto al tiempo de servicio de los docentes de la institución educativa San Joaquín, del total de 30 encuestados, el 16,7\% señaló que labora menos de 2 años, el $20 \%$ entre 2 y 5 años, 36,7\% marcaron el rango 5-8.

Respecto a la Variable: Comunicación interna, la información se presentará por dimensiones: Patrones de comunicación, barreras de la comunicación y retroalimentación. La dimensión patrones de comunicación se establecen a través de los indicadores cantidad de información y tipo de información. Respecto a la percepción de la dimensión Patrones de comunicación, el 43,3\% considera que "A veces" se cumple con los indicadores, mientras que la opción más marcada (50\%) es "casi siempre" y que la opción menos marcada es "siempre".

Respecto a la dimensión: Barreras de la comunicación, el 46,7\% considera que "A veces" se cumple con los indicadores, mientras que la opción más marcada $(53,3 \%)$ es "casi siempre.

Respecto a la percepción de la dimensión: Retroalimentación, el 43,3\% considera que "A veces" se cumple con los indicadores, mientras que la opción más marcada $(50 \%)$ es "casi siempre. Por otro lado, la opción menos marcada es "siempre" con $6,7 \%$.

Respecto a la Variable: Clima laboral, la información se presentará por dimensiones: Liderazgo, Relaciones interpersonales, Participación, Reconocimiento y Comunicación.

La dimensión: Liderazgo se establecen a través de los indicadores "Existe un ambiente de confianza entre compañeros" y "Se les permite a los docentes dar propuestas para mejorar el trabajo". Respecto a la percepción de la dimensión Liderazgo, el 40\% considera que "A veces" se cumple con los indicadores, mientras que la opción más marcada (60\%) es "siempre".

La dimensión: Relaciones interpersonales se establecen a través de tres indicadores: Compañerismo, Trabajo en equipo y Relaciones interpersonales. Respecto a la percepción de la dimensión Relaciones interpersonales, el $40 \%$ considera que "A veces" se cumple con los indicadores, mientras que la opción más marcada (60\%) es "siempre".

La dimensión: Relaciones interpersonales se establecen a través de tres indicadores: Colaboración, Participación e Integración. 


\section{Ciencia y Educación (ISSN 2707-3378) \\ Vol. 1 No. 2 \\ Febrero del 2020}

Respecto a la percepción de la dimensión Participación, el 43,3\% considera que "A veces" se cumple con los indicadores, mientras que la opción más marcada $(56,7 \%)$ es "siempre".

La dimensión: Reconocimiento se establecen a través de dos indicadores: "La mayoría de los trabajadores conoce las metas laborales" y "Existe integración y cooperación entre los miembros de la institución". Respecto a la percepción de la dimensión Reconocimiento, el $73,3 \%$ considera que "A veces" se cumple con los indicadores, mientras que la opción más marcada (26,7\%) es "siempre".

La dimensión Relaciones interpersonales se establecen a través de tres indicadores: Comunicación fluida, apertura a la búsqueda del bien común y alternativas de solución. Respecto a la percepción de la dimensión Reconocimiento, el 36,7\% considera que "A veces" se cumple con los indicadores, mientras que la opción más marcada $(63,3 \%)$ es "siempre".

Para evaluar la correlación entre variables categóricas ordinales, se puede emplear dos coeficientes: El chi cuadrado y Spearman, para la presente investigación se va a descartar la prueba chi cuadrado ya que requiere que en la tabla cruzada de las variables a evaluar, la frecuencia sea mayor a 5, y en nuestro caso no se cumple. Por tanto, se empleará el coeficiente de correlación de Spearman porque es una prueba no paramétrica, es decir, libre de distribución probabilística.

Respecto a la relación entre la comunicación interna y el clima laboral se observa que existe una relación entre moderada y fuerte con significación estadística. Esta correlación es positiva, es decir a mayor frecuencia con respecto a la comunicación interna, mayor satisfacción habrá en el clima laboral. $(\mathrm{p}<0.05)$

\section{Conclusiones}

El objetivo principal de la investigación fue establecer la relación que existe entre la comunicación interna se relaciona con el clima laboral de los docentes de la Unidad Educativa San Joaquín, de la Parroquia Cumbe, en el periodo 2018.

Comunicación interna y clima laboral. Se observó que el $30 \%$ considera que "A veces" se cumple con el clima laboral y la comunicación interna. Mientras que el 53,3\% considera "casi siempre" se establece la relación en ambas variables.

Según la prueba de hipótesis, se concluye que existe una relación entre moderada y fuerte con significación estadística. Esta correlación es positiva, es decir a mayor frecuencia con respecto a la comunicación interna, mayor satisfacción habrá en el clima laboral. $(\mathrm{p}<0.05)$

La comunicación interna en el área de patrones de comunicación y el clima laboral. Se observó que el $30 \%$ considera que "A veces" se cumple con el clima laboral y los patrones de comunicación. Mientras que el 50\% considera "casi siempre" se establece la relación en ambas variables. Así mismo, el 6,7\% optó por la categoría "siempre".

Según la prueba de hipótesis, se concluye que existe una relación entre moderada y fuerte con significación estadística. Esta correlación es positiva, es decir a mayor frecuencia con respecto a los patrones de comunicación, mayor satisfacción habrá en el clima laboral. $(\mathrm{p}<0.05)$

La comunicación interna en el área de barreras de la comunicación y el clima laboral. Se observó que el $30 \%$ considera que "A veces" se 


\section{Ciencia y Educación (ISSN 2707-3378) \\ Vol. 1 No. 2 \\ Febrero del 2020}

cumple con el clima laboral y las barreras de comunicación. Mientras que el 53,3\% considera "casi siempre" se establece la relación en ambas variables.

Según la prueba de hipótesis, se concluye que existe una relación entre moderada y fuerte con significación estadística. Esta correlación es positiva, es decir a mayor frecuencia con respecto a las barreras de comunicación, mayor satisfacción habrá en el clima laboral. $(\mathrm{p}<0.05)$

La comunicación interna en el área de retroalimentación y el clima laboral. Se observó que el $30 \%$ considera que "A veces" se cumple con el clima laboral y la retroalimentación. Mientras que el 53,3\% considera "casi siempre" se establece la relación en ambas variables.

Según la prueba de hipótesis, se concluye que existe una relación entre moderada y fuerte con significación estadística. Esta correlación es positiva, es decir a mayor frecuencia con respecto a la retroalimentación, mayor satisfacción habrá en el clima laboral. $(\mathrm{p}<0.05)$

\section{Recomendaciones}

Realizada la investigación, se sugieren las siguientes recomendaciones:

- Se sugiere al personal directivo y docentes desarrollar actividades deportivas o recreativas para generar un ambiente de confianza entre los compañeros de trabajo.

- Al personal directivo y a los docentes, se les sugiere que se fomenten foros de participación interna y externa para generar y mejorar la comunicación interna.

- A través de medios de difusión como periódico mural o memorandos informar por escrito por cambios o la información trascendental para la institución educativa.

- Cada coordinador del área debe proporcionar toda la información necesaria para realizar el trabajo, coordinar las tareas, transmitir información para la mejora del desempeño profesional docente.

- Al personal directivo se le sugiere informar de los objetivos, misión, visión y políticas de la institución educativa a todo trabajador nuevo de la comunidad educativa.

- Al personal directivo y coordinar de cada área, se les recomienda asignar obligaciones y responsabilidades, con claridad y objetividad, al personal de la comunidad educativa. Esta información debe ser a través de un medio tangible y con fecha objetivas para lograr las metas propuestas.

\section{Referencias bibliográficas}

Álvarez, A. (2011). Medición y evaluación en comunicación. Málaga: Instituto de Investigación en Relaciones Públicas.

Álvarez, C. E. M. (2006). Clima organizacional en Colombia: El IMCOC, un método de análisis para su intervención. Bogotá: Universidad del Rosario.

Andrade, H. (2005). Comunicación organizacional interna: proceso, disciplina y técnica. Madrid: Netbiblo.

Arizcuren, A., Cabezas, E., Cañeque, N., Casado, M., Fernández, P., Lacasta, J. y Soria, L. (2008). Guía de buenas prácticas de comunicación interna. Madrid: FEAPS.

Baladezo Toro (2014) La comunicación organizacional y su incidencia en el desarrollo organizacional de la empresa San Miguel Drive. [Tesis de Titulación]. Facultad de Ciencias Administrativas, Universidad Técnica de Ambato.

Bejarano Carbajal (2017) Influencia de la comunicación interna en el clima laboral de la plana docente, Institución educativa Mis primeras huellitas Chimbote - 2017. [Tesis de licenciatura]. Facultad de ciencias de la comunicación, Universidad César Vallejo.

Cabrera Yépez, María (2012) Gestión educativa en el fortalecimiento de la comunicación interna en el centro educativo Novus en la ciudad Guayaquil año 2012. Propuesta: Diseño e implementación de una guía metodológica de orientación y mediación de 


\section{Ciencia y Educación (ISSN 2707-3378) \\ Vol. 1 No. 2 \\ Febrero del 2020}

gestiones educativas para docentes. [Tesis de Maestría]. Facultad de Filosofía, Letras y Ciencias de la Educación, Universidad de Guayaquil. Recuperado de http://repositorio.ug.edu.ec/bitstream/redug/ 3690/1/Gesti\%C3\%B3n\%20educativa\%20e n\%20el\%20fortalecimiento $\% 20 \mathrm{de} \% 201 \mathrm{a} \% 2$ 0comunicaci\%C3\%B3n\%20integral\%20en $\% 20$ el $\% 20$ centro\%20educativo $\% 20$ Novus $\% 20$ de\%20Guayaquil\%202012.pdf

Castillo Dávila, Natalia (2014) Clima, motivación intrínseca y satisfacción laboral en trabajadores de diferentes niveles jerárquicos. [Tesis de licenciatura]. Universidad Católica del Perú, Lima.

Cervera, Á. L. (2008). Comunicación total. Madrid: Esic.

De Castro, A. (2017). Manual práctico de comunicación organizacional. Madrid: Editorial Verbum.

Dirección Nacional de Estándares Educativos (2017) Anexo 1: Estándares de Gestión Escolar y desempeño profesional directivo y docente.

Duque, P. C. (2005). La Comunicación y la gestión de la información en las instituciones educativas. México: WK Educación.

Elías, J. (2004). Experiencias sobre Sistemas de Incentivos a la Comunidad de Investigadores para Fortalecer la Innovación Tecnológica. Colombia: Instituto Interamericano de Cooperación para la Agricultura-IICA.

Enrique, A. M. (2008). La planificación de la comunicación empresarial (Vol. 202). Univ. Autónoma de Barcelona.

Equipo Vértice. (2007). Comunicación interna. Málaga: Editorial Vértice.

Fernández Collado, C., \& Galguera García, L. (2008). La comunicación humana en el mundo contemporáneo (No. 302.2 F363c). México: McGraw Hill.

Fernández-Ríos, M., \& Sánchez, J. C. (1997). Eficacia organizacional: concepto, desarrollo y evaluación. Madrid: Ediciones Díaz de Santos.

Fernández Verde, D. O. L. O. R. E. S., \& Fernández Rico, E. L. E. N. A. (2010). Comunicación empresarial y atención al cliente. Madrid, España: Paraninfo.
Fiallos Núñez, L. A. (2012). La Comunicación Interna y su efecto en el Desempeño Laboral de la Institución SECAP de Ambato (Bachelor's thesis). Ecuador: Universidad Técnica de Ambato.

Govea, M., Domínguez, M. M. E., \& San Agustín, Y. Q. (2012). Importancia del clima laboral en los resultados de una empresa y la competitividad. Contribuciones a la Economía, (2012-11).

González, M.; Nieto, J.; Portela, A. (2003) Organización y Gestión de Centros Escolares: dimensiones y procesos. Madrid, Pearson Educación

Guerola Salinas, Milagros (2018) Análisis de la comunicación interna en la institución educativa CEDEUNSA Arequipa, 2017. [Tesis de grado] Escuela Profesional de Comunicación Social, Universidad Católica de Santa María, Arequipa. Recuperado de http://tesis.ucsm.edu.pe/repositorio/bitstrea $\mathrm{m} /$ handle/UCSM/7451/47.1103.CS.pdf?seq uence $=1 \&$ isAllowed $=\mathrm{y}$

Guato Rojas, M. L. (2013). La comunicación interna y el ambiente laboral en el Colegio Técnico Provincia de Pastaza de la ciudad de Puyo. Ecuador: Universidad Técnica de Ambato.

Guzmán, V. (2012). Comunicación organizacional. México: Milenio.

Kouss Gutiérrez, S. (2015). La comunicación interna en las organizaciones. España: Gredos.

Llaneza, F. J. (2007). Ergonomía y psicosociología aplicada: manual para la formación del especialista. España: LEX NOVA.

Noboa, A. (2007). Especificidades del clima organizacional en las instituciones de salud. Macau: Universidad de la República de Uruguay.

Ongallo, C. (2007). Manual de comunicación: guía para gestionar el conocimiento, la información y las relaciones humanas en empresas y organizaciones. España: Librería-Editorial Dykinson.

Osorio Madero, Santiago (2009) "La función de la comunicación interna y externa, como instrumento estratégico para mejorar el servicio al cliente en Madero y Maldonado, 
corredores de seguros" [Tesis de grado] Facultad de Comunicación y lenguaje, Pontificia Universidad Javeriana, Colombia. Recuperado de http://javeriana.edu.co/biblos/tesis/comunic acion/tesis293.pdf

Pérez, G., Pineda, U., y Arango, M. D. (2011). La capacitación a través de algunas teorías de aprendizaje y su influencia en la gestión de la empresa. Revista Virtual Universidad Católica del Norte, 1 (33), 1-22.

Ramos Palacios, Wilder Fabio (2015). Los movimientos sociales en la web 2.0: La construcción de la opinión pública sobre la protesta social \#NoAlaRepartija. [Tesis de Maestría]. Facultad de Ciencias Sociales, Universidad Nacional Mayor de San Marcos.

Reyes Bedriñana, Magno (2017) El clima laboral y la motivación de los DocentesPersonal administrativo del Nivel Primaria de la I.E. Pedro del Castillo Ríos- ex 160 de la ciudad de Yurimaguas, 2015 [Tesis de Maestría] Escuela de Posgrado, Universidad César Vallejo, Lima. Recuperado de http://repositorio.ucv.edu.pe/bitstream/handl e/UCV/986/reyes_bm.pdf?sequence $=1$

Reyes, J. (2012). Las Cuatro Dimensiones de la Comunicación Interna. Cuadernos del Centro de Estudios en Diseño y Comunicación. Ensayos, (40), 127-138.
Romero Sornoza (2013) Plan de Comunicación interna y externa para la Universidad Internacional del Ecuador Extensión Guayaquil. [Trabajo de titulación] Facultad de Ciencias Sociales y Comunicación, Universidad Internacional del Ecuador.

Vega, J. C., Díaz, E. G. R., y Montoya, A. (2012). Metodología de evaluación del clima organizacional a través de un modelo de regresión logística para una universidad en Bogotá, Colombia. Revista CIFE: Lecturas de Economía Social, 14(21), 63.

Wills-Espinosa, N., Cevallos Icaza, M., Sadi, G. y Ancin I. (2017). La relación entre la satisfacción con la comunicación interna y el compromiso organizacional: el caso de una universidad ecuatoriana. Austral comunicación, 6 (1), pp. 133-160. Recuperado de http://www.austral.edu.ar/ojs/index.php/aust ralcomunicacion/article/view/182

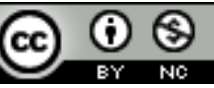
Creative Commons Reconocimiento-No Comercial 4.0 Internacional. Copyright (c) Henry Patricio Iñaguazo Macas 